\title{
Observation of energy-time dispersed ion structures in the magnetosheath by CLUSTER: possible signatures of transient acceleration processes at shock
}

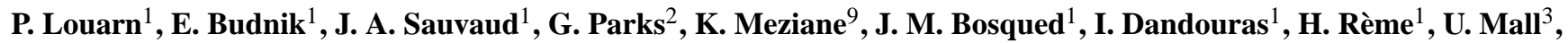 \\ P. Daly ${ }^{3}$, M. Dunlop ${ }^{4}$, A. Balogh ${ }^{5}$, L. M. Kistler ${ }^{6}$, and E. Amata ${ }^{7}$ \\ ${ }^{1}$ CESR, Toulouse, France \\ ${ }^{2}$ University of California, Berkeley, CA, USA \\ ${ }^{3}$ MPAe, Lindau, Germany \\ ${ }^{4}$ Rutherford Appleton Laboratory, UK \\ ${ }^{5}$ Blackett Laboratory, Imperial College, London, UK \\ ${ }^{6}$ University of New Hamsphire, Durham, USA \\ ${ }^{7}$ ISFI, Rome, Italy
}

Received: 15 October 2002 - Revised: 30 January 2003 - Accepted: 11 February 2003

\begin{abstract}
We analyse energy-time dispersed ion signatures that have been observed by CLUSTER in the dayside magnetosheath. These events are characterized by sudden increases in the ion flux at energies larger than $10 \mathrm{keV}$. The high energy ions $(30 \mathrm{keV})$ are first detected, with the transition to the low energy ions ( $5 \mathrm{keV}$ ) lasting about $100 \mathrm{~s}$. These injections are often associated with transient plasma structures of a few minutes in duration, characterized by a hotter, less dense plasma and a diverted flow velocity, thus presenting similarities with "hot flow anomalies". They also involve modifications of the magnetic field direction, suggesting that the shock interacts with a solar wind discontinuity at the time of the event. The injections can originate from the magnetosphere or the shock region. Studying in detail a particular event, we discuss this last hypothesis. We show that the observed energy/time dispersion can be explained by combining a time-of-flight effect with a drift of the source of energetic particles along the shock. We propose that the acceleration results from a Fermi process linked to the interaction of the discontinuity with a quasi-perpendicular shock. This model explains the observed pitch-angle selection of the accelerated particles. The Fermi process acting on the beam of ions reflected from the shock appears to be sufficiently efficient to accelerate over short time scales (less than $30 \mathrm{~s}$ ) particles at energies above $30 \mathrm{keV}$.
\end{abstract}

Key words. Magnetospheric physics (solar-wind-magnetosphere interaction; magnetosheath) - Space plasma physics (shock waves)

Correspondence to: P. Louarn

(Philippe.Louarn@cesr.fr)

\section{Introduction}

Energy-time dispersed particle signatures are commonly observed in the magnetosphere. These phenomena (injections) have been detected by a number of spacecraft in regions as different as the inner magnetosphere, the near and distant magnetotail, the cusp and the flanks (Arnoldy and Chang, 1969; DeForest and McIlwain, 1971; Konradi et al., 1975; Carlson and Torbert, 1980; Sauvaud et al., 1981; Mauk and Meng, 1983; Sandahl et al., 1998). Injections are characterized by sudden increases in the flux of particles with energies significantly larger than the ambient thermal energy, with the high energy particles being the first detected. The energy/time dispersion is generally interpreted as a time-offlight effect taking place between the site of injection and the observer. These signatures reveal the existence of localized and transient releases of energetic particles in the medium. Thus, they give information on the spatial/temporal development of energetic processes and help to remotely understand their physics (see, for example, Stenuit et al., 2001).

To our knowledge, the observations of energy-time dispersed signatures in the magnetosheath have never been reported. As discussed here, during its first months of operations, the CLUSTER ion spectrometers have detected a few tens of injections in the dayside magnetosheath, at distances larger than 12 Earth radii $\left(R_{E}\right)$ from Earth. The first goal of the paper is to present these new phenomena. It seems difficult to interpret magnetosheath injections by processes similar to those invoked for explaining magnetospheric injections. Their origin has thus to be elucidated. Close to boundary layers, a magnetic connection with the magnetosphere is not excluded. In such a case, the possible magnetospheric origin of the dispersed structures has to be con- 
sidered. Farther from Earth, the characteristics of the magnetosheath plasma are mainly determined by shock-related thermalization processes. One is then inclined to relate the observed injections to transient modifications in the heating/acceleration regime at shock. For reasons presented later, we will focus our discussion on this last hypothesis.

The fact that transitory phenomena may develop at collisionless shocks has been the subject of numerous studies. These phenomena may have a pure internal origin and be linked to the physics of the shock itself. For example, quasiparallel shocks are characterized by large-amplitude electromagnetic waves propagating in both the upstream and downstream regions. When interacting with the shock, they may disrupt the front of the shock that thus present cycles of disruption/re-formations with a typical periodicity of a few minutes (Greenstadt et al., 1982). As shown by numerical simulations, sequences of disruption/re-formation modulate the plasma characteristics and produce alternating cold and hot ion regions downstream of the shock (Burgess, 1989a; Thomas et al., 1990; Winske et al., 1990). On the other hand, external disturbances may also be the cause of transitory phenomena. When solar wind discontinuities (as current layers) collide the shock, particularly the heating/acceleration mechanisms may develop and modify the plasma characteristics over short time scales, both upstream and downstream of the shock. The "Hot Flow Anomalies" (HFA) are typical examples of such transient events (see the review by Schwartz, 1995). These phenomena observed close to the shock correspond to the formation of regions of hot, tenuous and less magnetized plasma over a time scale of a few minutes. As demonstrated by Sibeck et al. $(1998,1999)$, they may significantly modify the magnetopheric boundary conditions, leading to radial displacements of the magnetopause over distances as large as $5 R_{E}$.

By studying in detail a particularly well-defined dispersed structure observed close to the shock as CIS was operating in burst mode; we show that it very likely corresponds to another type of transitory phenomena developing at shocks. The second goal of the paper will be to identify the cause of these modifications and to describe the heating/acceleration underlying process (Sects. 3 and 4). We propose that they are related to a particular interaction between a quasi-perpendicular shock and a solar wind discontinuity that triggers a burst of efficient ion Fermi acceleration.

\section{Examples of transient signatures observed in the magnetosheath}

In Fig. 1, we show $40 \mathrm{~min}$ of observations performed in the magnetosheath by the ion spectrometer (CIS experiment, Rème et al., 1997) and the flux gate magnetometer (FGM experiment, Balogh et al., 1997) on 10 March 2001. The observation is made at $14.4 R_{E}$ from Earth, well above the equatorial plane $\left(Z_{\mathrm{GSE}}=8.9 R_{E}\right)$ and near the Sun/Earth meridian plane $\left(Y_{\mathrm{GSE}}=1.8 R_{E}\right)$. In the two upper panels, the CLUSTER trajectory is plotted in GSE frame. For refer- ence, we indicate the possible positions of the magnetopause and the shock in the $Z=0$ and $Y=0$ planes for solar wind conditions corresponding to $B_{z}=-2 \mathrm{nT}$ and $P_{s w}=1 \mathrm{nPa}$ (Shue et al., 1998). For typical solar wind conditions, CLUSTER would be thus well inside the magnetosheath, at almost equal distance from the magnetopause and the shock. The six panels presented below show, respectively, (from the top panel): (1) the ion energy/time spectrogram in the range $1 \mathrm{keV}-30 \mathrm{keV}$, (2) the density, (3) the three components of the velocity, (4) the ion temperature (parallel and perpendicular to the magnetic field), (5) the magnetic field measured by FGM. In the last panel, the magnetic field measured by ACE far upstream in the solar wind is presented. It is plotted, taking into account a time delay of $2840 \mathrm{~s}$. This delay has been estimated from the solar wind velocity measured by ACE and adjusted to optimize the correlation between the magnetic fluctuations observed by ACE and CLUSTER.

This figure displays an overview of a dynamic magnetosheath, characterized by the juxtaposition of different magnetosheath plasma states and disturbances (for a review on magnetosheath, see Song and Russell, 1999). This dynamical character has to be related to the fact that at least three solar wind discontinuities collide with the magnetosphere during the time of observation. Two of them, at 08:47 and 09:03 UT, are sudden rotations of the interplanetary magnetic field (IMF). A more complex signature is observed around 08:29 UT, during a perturbed period lasting until 08:35 UT. Nevertheless, despite these disturbances, steady states are observed from 08:35 to 08:44 UT and from 08:48 to 09:01 UT. They correspond to a laminar flow presenting only small fluctuations in density, velocity, temperature and magnetic field (fluctuations smaller than $20 \%$ of the averaged values). It is also characterized by a pronounced temperature anisotropy (perpendicular temperature larger than the parallel one) and small amplitude (a few $\mathrm{nT}$ ) compressional disturbances. These plasma states are typical of the region downstream from a quasi-perpendicular shock (Crooker and Siscoe, 1977; Hubert et al., 1989; Lacombe et al., 1992; Fazakerley and Southwood, 1994). Before 08:32 UT, around 08:46 and after 09:00 UT, significantly more turbulent states are observed. They are characterized by large fluctuations in density, temperature and magnetic field (fluctuations reaching $100 \%$ of the averaged values), a reduced temperature anisotropy and a larger flux of particles above $10 \mathrm{keV}$. These are some of the characteristics of a plasma downstream from a quasi-parallel shock (Greenstatd et al., 1972; Kennel et al., 1984; Scudder, 1984; Entgebretson et al., 1991). However, given the unsteady character of the solar wind and the arrival of current discontinuities on the shock, other interpretations than transitions from quasi-perpendicular to quasi-parallel geometry have to be considered.

The turbulent state observed before 08:32 most probably corresponds to the crossing of a boundary layer. Before 08:25 UT (data not shown in the figure), CLUSTER has indeed entered into the magnetosphere for a short period of time from 08:10 to 08:15 UT. From 08:15 to 08:32 UT, it then stays in a turbulent region where energetic oxygen ions 
SAMBA (SC-3)

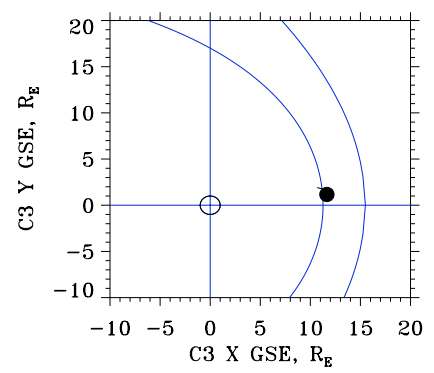

10 March 2001

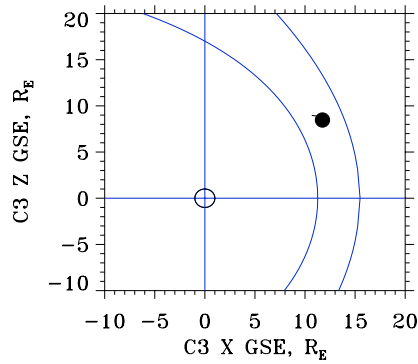

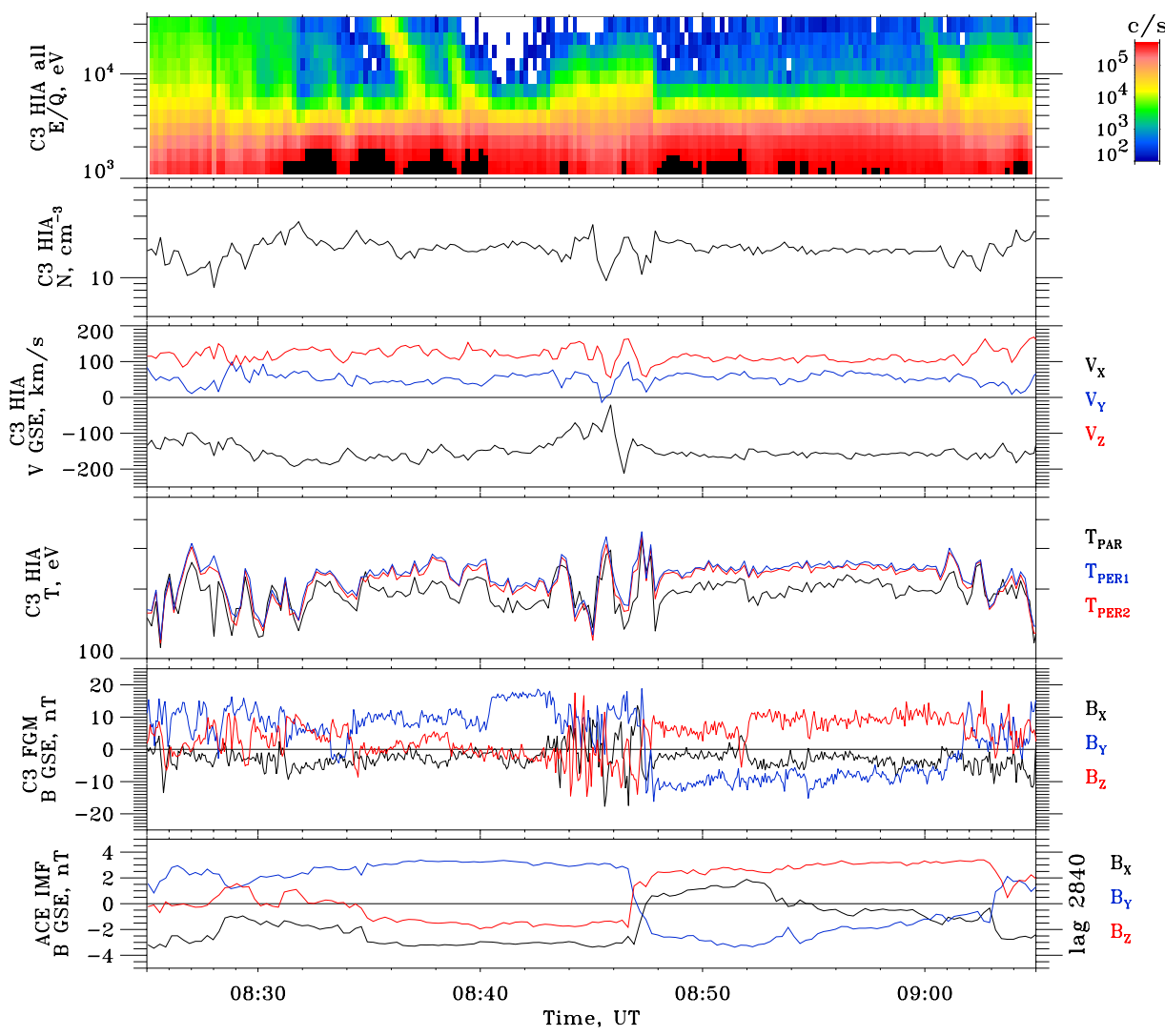

Fig. 1. Example of transient phenomena observed in the magnetosheath by CLUSTER (10 March 2001). The CLUSTER position, the magnetopause and the shock are shown in the two upper plots. Data are presented below, from top to bottom: (1) Energy/time spectrogram from 2 to $30 \mathrm{keV}$ measured by CIS, (2) density, (3) plasma velocity, (4) parallel and perpendicular temperatures, (5) magnetic field measured by FGM and, (6) magnetic field measured by ACE far upstream in the solar wind. It is shown with a delay of $2840 \mathrm{~s}$.

are detected which indicates that CLUSTER is magnetically connected to the magnetosphere.

The transient structure observed from 08:44 to 08:48 UT is of a different nature. It can be assimilated to a turbulent plasma state embedded in two laminar flows. The flux of energetic particles (above $20 \mathrm{keV}$ ) is smaller than the one observed in the boundary layer and no oxygen ions is detected. CLUSTER is thus well inside the magnetosheath during this time period. As seen in panel (5), the turbulent zone is associated with a magnetic field rotation. Before 08:44 UT, the main magnetic component is in the $Y$ direction $\left(B_{y} \sim 18 \mathrm{nT}\right)$. From 08:44 to 08:48 UT, the $Y$ component re- verses and a positive $B_{z}$ appears, the final magnetic field being: $B_{y} \sim-10 \mathrm{nT}, B_{z} \sim 8 \mathrm{nT}$. ACE observations suggest that this rotation is related to the interaction of the shock with a solar wind discontinuity. The signature is characterized by strong anti-correlated density and temperature fluctuations (from 10 to more than $20 \mathrm{~cm}^{-3}$ and $150 \mathrm{eV}$ to $250 \mathrm{eV}$ ) and large velocity deviations. In particular, the $V_{x}$ component is strongly reduced (from $-150 \mathrm{~km} / \mathrm{s}$ to $-70 \mathrm{~km} / \mathrm{s}$ ). The magnetic field is variable with a decrease in its averaged magnitude (from $20 \mathrm{nT}$ to less than $10 \mathrm{nT}$ ).

This transient phenomenon presents many similarities with "hot flow anomalies" (HFA). As described by a num- 


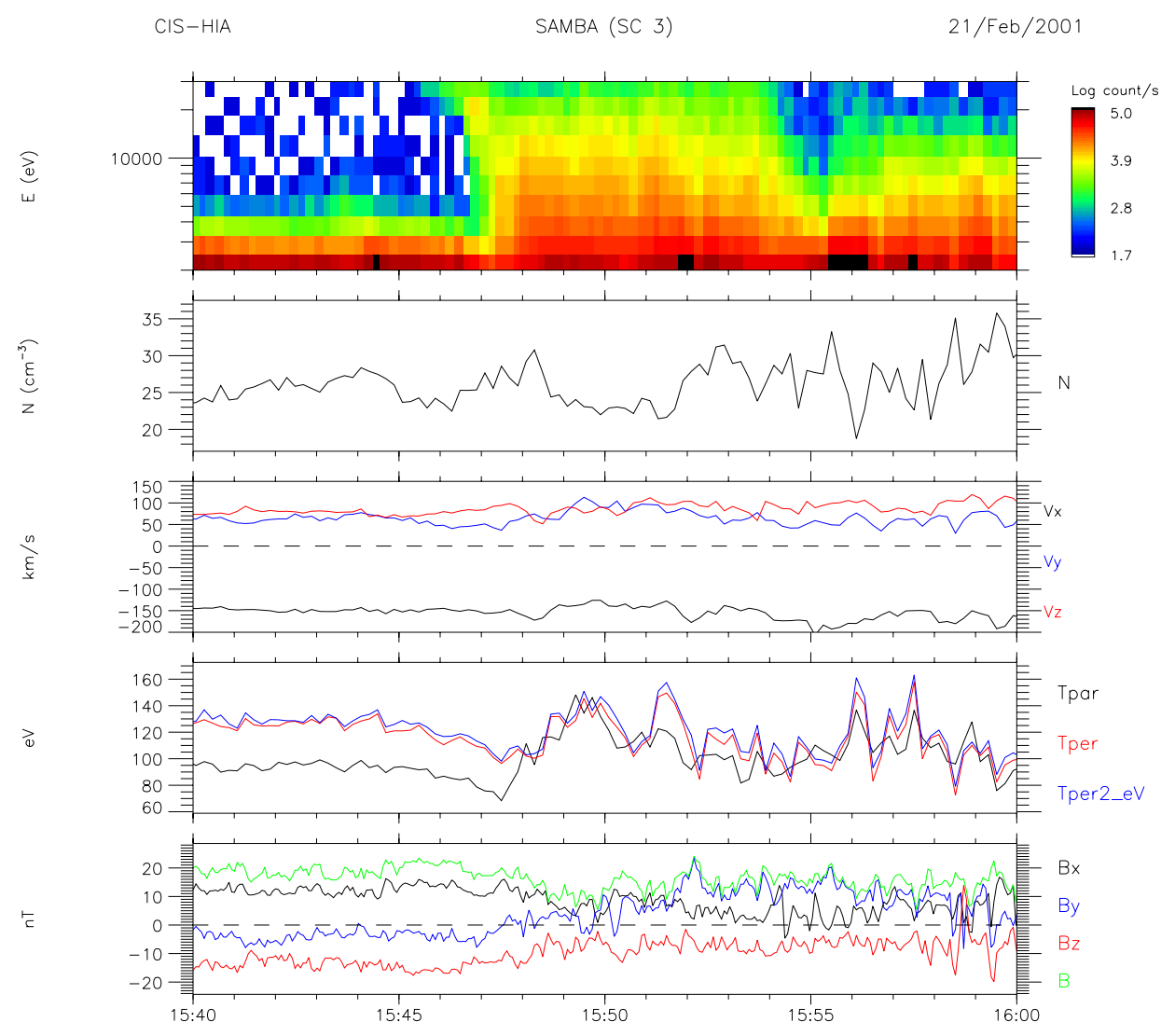

Fig. 2. Dispersed structure and associated HFA observed on day 21 February 2001. From top to bottom: (1) Energy/time spectrogram from 2 to $30 \mathrm{keV}$, (2) density, (3) plasma velocity, (4) parallel and perpendicular to the magnetic field temperature and (5) magnetic field measured by FGM.

ber of authors (Schwartz et al., 1985; Thomsen et al., 1986; Paschmann et al., 1988; Schwartz et al., 1988; Thomsen et al., 1988; Sibeck et al., 1999), these phenomena, when observed close to the shock, are interpreted as 3-dimensional bubbles of hot plasma that form a bulge on the shock. The core of HFA is a hot, tenuous, low magnetized plasma with a flow velocity deflected tangentially to the shock. They would result from the interaction of the shock with solar wind current sheets (see the models proposed by Burgess, 1989b; Thomsen et al., 1993). The downstream signature of HFA remains relatively unclear, although phenomena that have been assimilated to such signatures are presented and discussed by Schwartz et al. (1985), Thomsen et al. (1986) and Paschmann et al. (1988). These reported events are very comparable to the one presented here. Thus we interpret the transient structure seen around 08:46 UT as a HFA-type phenomena. The turbulent plasma observed after 09:01 UT could also be associated to similar phenomena. We will not study it in detail here, in order to concentrate on another type of phenomena.

One distinguishes a second type of transient signature around 08:36, 08:38 UT and, although less visible, 09:01 UT. They are shorter (1-min duration) and are characterized by a clear energy-time dispersion. These dispersed signatures (MDS for Magnetosheath Dispersed Signatures, hereafter) correspond to the sudden detection of protons with energy larger than $5 \mathrm{keV}$. They are first observed at the highest energy range ( $30 \mathrm{keV}$ here), with the transition from the high to the low energies lasting $\sim 1-2 \mathrm{~min}$. The flux increase can be considerable. For example, at 08:36 UT, the flux above $20 \mathrm{keV}$ is almost 2 orders of magnitude larger than the one associated with HFA. Oxygen ions are not detected in association with these signatures.

We estimate that more than twenty MDS have been detected for a period of two months (February/March 2001). They are generally not isolated. Thus, the example at 09:01 UT is detected during the transition from a steady to a more turbulent plasma state, which also corresponds to a rotation of the magnetic field that may have triggered the formation of an HFA. The two other examples (08:36 and 08:38 UT) occur in between the crossing of a boundary layer and a transient signature that was interpreted as being an HFA-type phenomenon. In Fig. 2, we present a very similar sequence of phenomenon. It is observed on 21 February 2001, at $X_{\mathrm{GSE}}=8.8, Y_{\mathrm{GSE}}=4.8$, and $Z_{\mathrm{GSE}}=9.1 R_{E}$. Twenty minutes of data are presented. The five panels shown in the figure are similar to the ones in Fig. 1. On a global scale, the magnetosheath is much more quiet than for previous example. Nevertheless, a rotation of the magnetic field occurs from 15:47 to 15:52 UT, with the $B_{y}$ component varying from $-3 \mathrm{nT}$ to $15 \mathrm{nT}$. This time period corresponds to a 
small but significant increase in temperature, and a decrease in density and deviations in velocity, which is again typical of a HFA-type phenomena. No oxygen ions are observed during this time period. ACE observations (not shown here) suggest that a solar wind perturbation could have collided with the shock during this time interval. Just before the crossing of this hot plasma region (before 15:47:30 UT), as CLUSTER is clearly downstream from a quasi-perpendicular shock, a dispersed structure is observed starting at 15:45:45 UT and lasting about $1 \mathrm{~min}$. The structure is seen at 8:36 UT in Fig. 1; it is characterized by a flux above $20 \mathrm{keV}$, significantly larger than the typical flux observed during the HFA (flux increase by a factor larger than 2 , in the present case).

From these observations, we conclude that HFA and MDS are somewhat related phenomena. MDS are indeed often observed in association with HFA-type phenomena and are thus likely to be related to the interaction of current discontinuities with the shock, which, however, does not necessarily mean that MDS particles come from the shock. For example, the first two examples seen in Fig. 1 are detected just a few minutes after CLUSTER exits a boundary region and their magnetospheric origins cannot be excluded. Conversely, the 09:01 example of Fig. 1 and the one of Fig. 2 are attached to transient signatures that may directly originate from the shock. For such cases, the most simple interpretation would be that MDS are, as HFA, linked to short scale modifications in the shock energization processes, due to the interaction with a solar wind perturbation. However, the nature of these modifications would be quite different: HFA indeed correspond to a plasma heating when MDS would be rather associated to an efficient ion acceleration up to energies larger than $10 \mathrm{keV}$. This point concerning the possible shock origin of MDS is analyzed in more detail in the next section.

\section{Detailed analysis of a particular case}

The dispersed structure presented in Fig. 3 has been observed on 28 January 2001 when CIS was operating in burst mode. Complete 3-D distribution functions are thus measured with a 4-s resolution. The format of the figure is similar to the one of Fig. 1. At the time of observation, CLUSTER is in the flank of the magnetosheath, well above the equatorial plane: $X_{\mathrm{GSE}}=5.1, Y_{\mathrm{GSE}}=7.45$ and $Z_{\mathrm{GSE}}=$ $9.25 R_{E}$. The event is first detected around 19:41:30 UT at $30 \mathrm{keV}$. Ninety seconds later, particles at $5 \mathrm{keV}$ are observed. The event itself lasts $\sim 80 \mathrm{~s}$ at a given energy. During the event, from 19:41:30 to $19: 43: 00 \mathrm{UT}$, the characteristics of the thermal plasma remain unchanged. The density is constant $\left(120 \mathrm{~cm}^{-3}\right)$, as well as the velocity $(-150 \mathrm{~km} / \mathrm{s}$, $80 \mathrm{~km} / \mathrm{s}, 50 \mathrm{~km} / \mathrm{s})$ and the temperature $(100 \mathrm{eV})$. As the signature merges with the ambient plasma (at 19:43:20 UT), a sudden transition in the plasma characteristics is observed. The density decreases from $120 \mathrm{~cm}^{-3}$ to $60 \mathrm{~cm}^{-3}$, the temperature increases from $100 \mathrm{eV}$ to more than $200 \mathrm{eV}$ and the flow velocity is deflected. The flow is first accelerated (from 19:43:20 to $19: 43: 40$ UT) with larger components in the $Y$ and $Z$ directions. The $X$ component (in antisolar direction) is then strongly reduced (from $-200 \mathrm{~km} / \mathrm{s}$ to $-80 \mathrm{~km} / \mathrm{s}$ ). Before the event, the magnetic field is very steady: $B_{x}=-3 \mathrm{nT}$, $B_{y}=20 \mathrm{nT}, B_{z}=5 \mathrm{nT}$. It presents large fluctuations (100\%) during the event and its mean amplitude is reduced (10 nT). Later (after 19:45), a steady field is again observed. It has nevertheless slightly rotated: $B_{x}=-6 \mathrm{nT}, B_{y}=25 \mathrm{nT}$, $B_{z}=5 \mathrm{nT}$. The MDS are thus detected just prior to the crossing of a disturbed plasma region that can be assimilated to a HFA-type phenomena. Note again that the observation is made downstream from a quasi-perpendicular shock. The sequence of phenomena is thus exactly similar to those already described. Let us also note that the observation is likely made not far from the shock, since it is crossed almost ten minutes later (around 19:53 UT).

The magnetic signature is more subtle than the ones observed previously. This time period is indeed very quiet. The time delay between ACE and CLUSTER would be of the order of $4160 \mathrm{~s}$. Generally, it is possible to optimize this delay by correlating structures seen by ACE and CLUSTER. In the present case, both the magnetic fields measured by ACE and CLUSTER show slow variations during the period of interest. The only clear structure propagating in the solar wind during the whole period is precisely the disturbance presented in the figure (at 19:41). It corresponds to a short scale rotation of the magnetic field. Before the crossing of the discontinuity, the magnetic field is mainly in the $y$ direction with a small $z$ component: $B=(-0.5 \mathrm{nT}, 2 \mathrm{nT}, 6 \mathrm{nT})$. In the discontinuity, the field rotates by almost $90^{\circ}$ to be in the $x / z$ plane $B=(4 \mathrm{nT}, 0.5 \mathrm{nT}, 5 \mathrm{nT})$. Since this unique structure detected by ACE is observed with a time delay consistent with the solar wind velocity, we propose that it is related to the transient structure seen by CLUSTER. To give credit to this interpretation, we will show now that the observed energy/time dispersion of the MDS can be explained if one considers that the injection site drifts along the shock at a velocity of the order of the typical solar wind velocity.

The starting point of our analysis concerns the nonclassical shape of the observed dispersion. In the case of a simple time-of-flight effect, the energy of the particles measured at time $t$ is:

$E(t)=\frac{m}{2}\left(\frac{L}{t}\right)^{2}$,

where $L$ is the distance between the source of particles and the observer. The logarithm of the energy thus varies as $-2 \log (t)$, with the second time derivative of the associated curve being then positive. The signature presented in Fig. 3 has a negative second derivative (see the details in Fig. 4). A more complex model than a simple time-of-flight must be used to account for this shape. As discussed below, this can be explained by a combination of time-of-flight effect, convection in the magnetosheath and drift of the injection site along the shock.

Let us consider the configuration displayed in Fig. 4 (top panel). The shock (in red) interacts with a discontinuity that 
SAMBA $(\mathrm{SC}-3)$

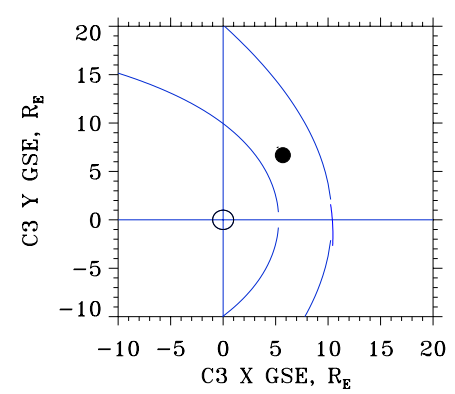

28 January 2001

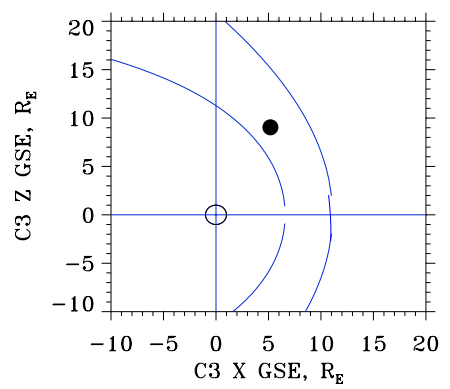

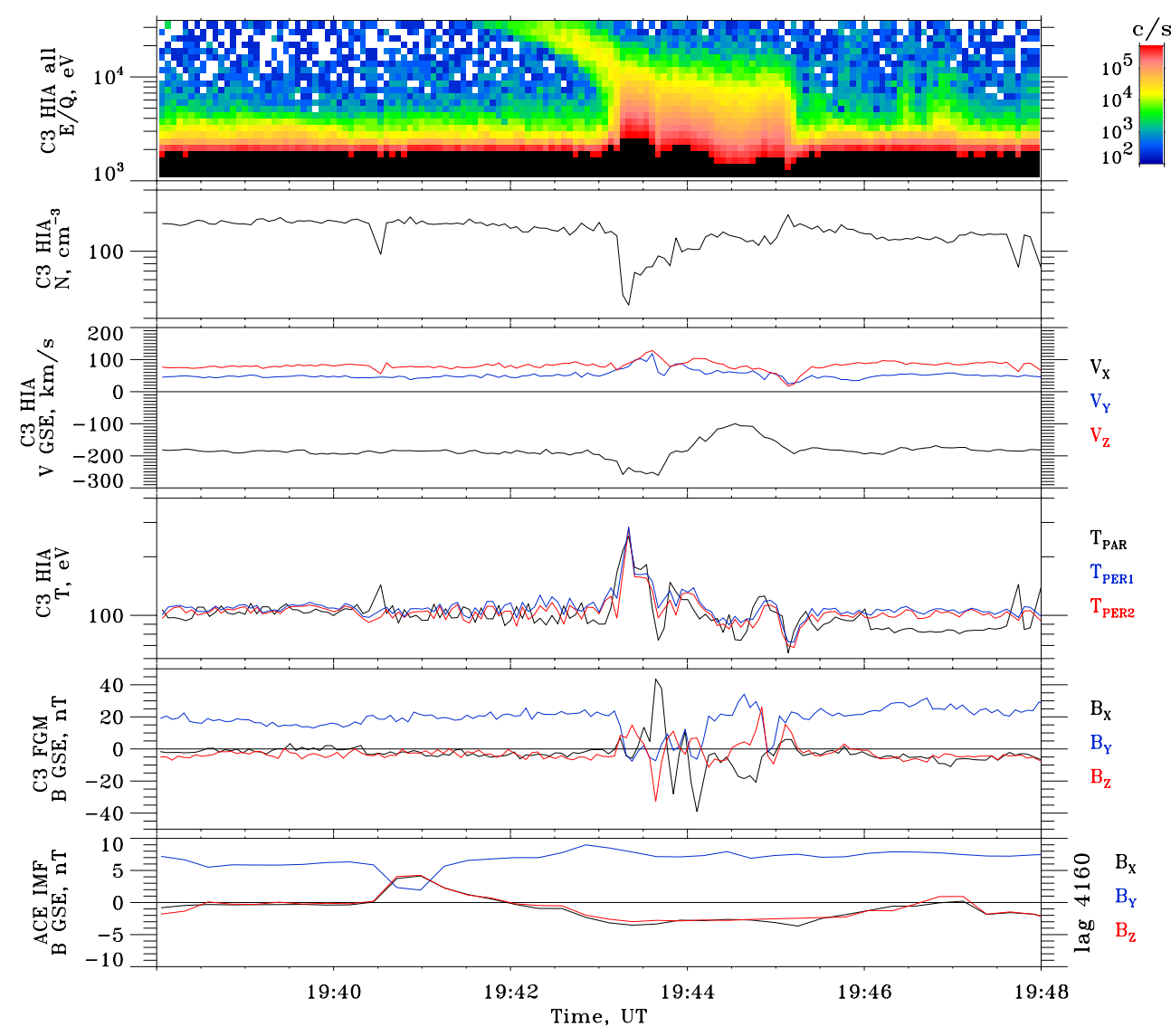

Fig. 3. Dispersed structure and associated HFA observed on day 28 January 2001. The CLUSTER position, the magnetopause and the shock are shown in the two upper plots. Data are presented below, from top to bottom: (1) Energy/time spectrogram from 2 to $30 \mathrm{keV}$ measured by CIS, (2) density, (3) plasma velocity, (4) parallel and perpendicular temperatures, (5) magnetic field measured by FGM and (6) magnetic field measured by ACE far upstream in the solar wind. It is shown with a delay of $4160 \mathrm{~s}$.

propagates in the solar wind (dashed black line). We assume that the energetic particles are injected into the magnetosheath from an interaction region corresponding to the intersection between the shock and the discontinuity. The source of energetic particles thus slides along the shock with a velocity $X$ component equal to the solar wind velocity: $V_{x}=V_{S W} \sim-300 \mathrm{~km} / \mathrm{s}([X, Y, Z]$ correspond to the classical GSE axis). In the figure, we present successive positions of the discontinuity. Once in the magnetosheath, the particles are convected at the local flow velocity $\left(V_{M S} \sim-120 \mathrm{~km} / \mathrm{s}\right)$ that is assumed to be in the $X$ direction only. No plasma deflection around the magnetosphere is considered. For a first approximation, we also consider that the magnetosheath magnetic field is uniform, in the $Y$ direction. $B_{Y}$ is indeed the main component measured by CLUSTER during this time period. We also assume that the particles have free dynamics along the magnetic field after their injection in the magnetosheath. Possible trajectories resulting from the convection (in $X$ direction) and the free dynamics (in $Y$ direction) are displayed. The difference between the model and the classical time-of-flight situation is linked to (1) the drift along the shock and thus, the variation of the distance between the in- 

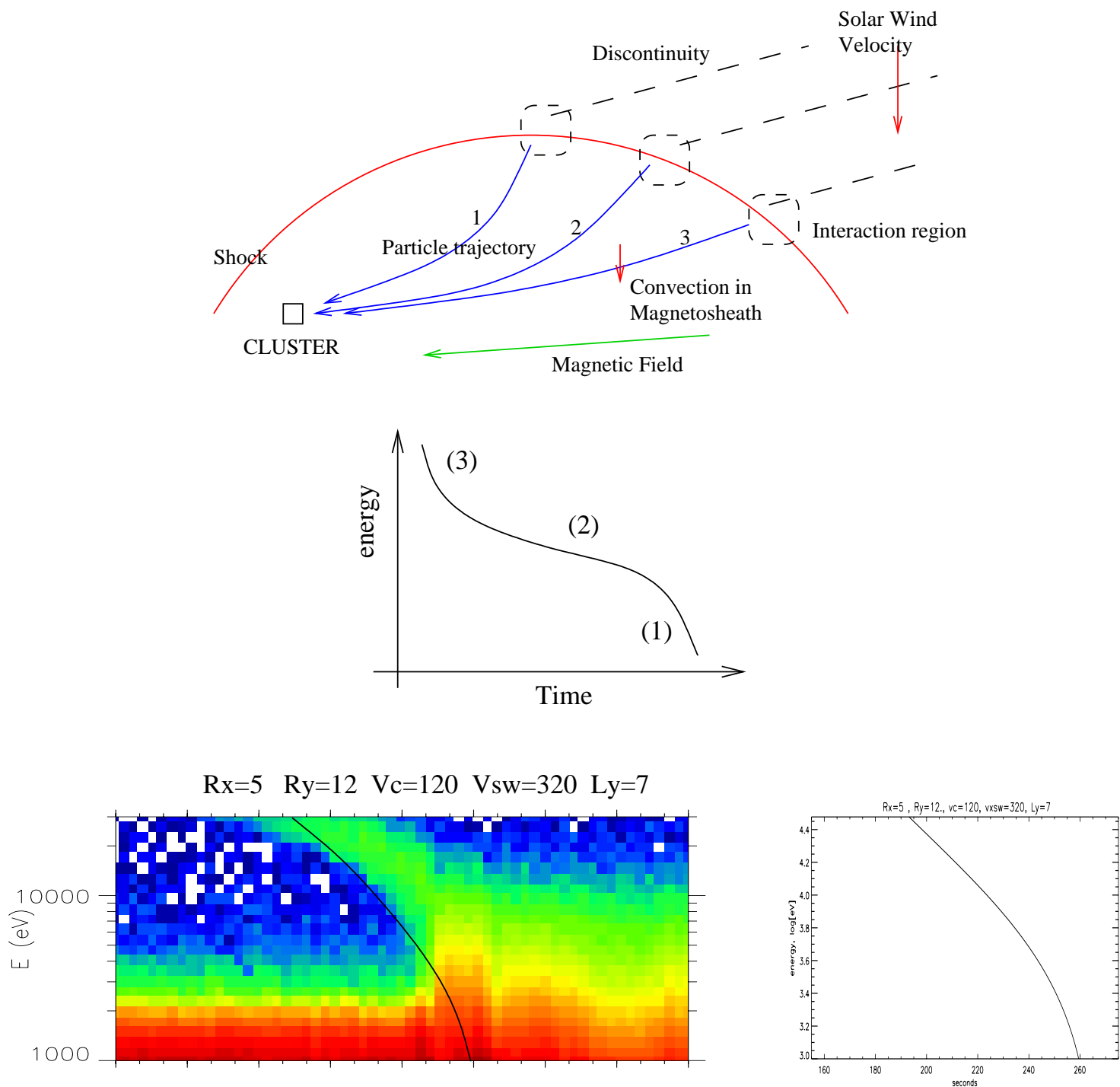

19:41

$19: 45$

Fig. 4. The cinematic model of the interaction. From top to bottom: (1) global configuration of the interaction and examples of trajectories, (2) typical dispersion calculated from the model and, (3) fit of the observations.

jection site and CLUSTER (particles injected recently travel over larger distances) and (2) the role of the convection (low energy particles are convecting and present almost no timeof-flight dispersion). Due to these different effects, the energy/time dispersion has a more complex shape than for simple time-of-flight. Let us consider a shock with a simple 2-D parabolic shape:

$$
X=X_{0}\left(1-\left(\frac{Y}{Y_{0}}\right)^{2}\right)
$$

The particle trajectory is described by two parametric curves:

$$
\begin{aligned}
& x(t)=X\left(t_{i}\right)+\mathrm{V}_{\mathrm{MS}}\left(t-t_{i}\right) \\
& y(t)=Y\left(t_{i}\right) \pm \sqrt{\frac{2 E}{m}}\left(t-t_{i}\right),
\end{aligned}
$$

where $t_{i}$ is the time of injection of the particle at shock, $X\left(t_{i}\right)$ and $Y\left(t_{i}\right)$ give the position of injection region. $X$ and $Y$ are related by Eq. (2). Let us note that Eq. (3b) describes a timeof-flight effect for particles with velocity aligned with the magnetic field. This corresponds to a minimal energy of the particle $\left(E=1 / 2 \mathrm{~m} \mathrm{~V}_{Y}^{2}\right)$. Assuming that the discontinuity reaches the shock at $t=0$, one has: $X\left(t_{i}\right)=X_{0}+V_{S W} t_{i}$. Putting $x(t)=0$ and $y(t)=L y$ in Eq. (3), one obtains the minimum energy of the particle reaching CLUSTER at time $t$ :

$E(t)=\frac{m}{2\left(t-t_{i}\right)^{2}}\left(L y \pm Y_{0} \sqrt{\frac{\left|V_{S W}\right| t_{i}}{X_{0}}}\right)^{2}$

with : $t_{i}=\frac{X_{0}+V_{M S} t}{V_{M S}-V_{S W}}$. 
In Eq. (4), the sign $+(-)$ corresponds to particles coming from the $Y<0(Y>0)$ side of the shock. A typical energy/time curve corresponding to Eq. (4) is presented in the middle panel of Fig. 4. Contrary to simple time-of-flight effect, the second time derivative of the curve is negative in the low energy range, below a few $10 \mathrm{keV}$, depending on the parameters of the model. The low energy particles have been injected as the discontinuity has begun to interact with the shock, they reach the observer mainly due to the convection (trajectory 1), with their dominant displacement being along $X$. At higher energies, the time-of-flight effect becomes dominant. The particles come directly from the flank of the shock (trajectory 3 ). This corresponds to the upper part of the curve that is similar to the one obtained with a simple time-of-flight effect. It is important to note that the model requires asymmetry to adequately explain the observations. In the present case, only the sign + is retained in Eq. (4), meaning that the particles reach CLUSTER with a $V_{y}<0$ velocity. The origin of the asymmetry is explained later. We will indeed show that the possible fermi acceleration mechanism resulting from the shock/discontinuity interaction acts preferentially on one side of the shock, depending on the sign of the $z$ component of the $B$ field at the discontinuity.

In the lower panel of the figure, we show a fit of the observed energy-time dispersion. It has been obtained from Eq. (4) with the following values of the parameters: $X_{0}=5 R_{E}, Y_{0}=12 R_{E}, V_{M S}=-120 \mathrm{~km} / \mathrm{s}, V_{S W}=$ $-320 \mathrm{~km} / \mathrm{s}$ and $L y=7 R_{E}$. All these values can be considered as reasonable. It would be certainly possible to obtain an even better fit by considering a 3-D geometry (3-D parabolic shape for the shock) which, for example, would explain why the most energetic ions are observed almost ten seconds before what is predicted by the simple 2-D model. The interesting point here is that our simple model accounts for the non-classical shape of the energy/time dispersion. This confirms that MDS result from the interaction of a solar wind discontinuity with the shock or, more precisely, that the energetic particles come from a localized source drifting along the shock at $V_{x} \sim V_{S W}$.

\section{Acceleration process}

The time-of-flight/convection model discussed above does not put particular constraints on possible acceleration mechanisms except that it implies that the acceleration site drifts along the shock. As discussed now, the analysis of the distribution function helps to pinpoint a possible scenario for the formation of the dispersed structure and the underlying acceleration process.

In Fig. 5, we display 2-D cuts of the distribution function of the MDS observed on 28 January 2001. The upper plots are pitch-angle presentations of the distributions. Blue (red) corresponds to minimum (maximum) values. The lower panels are cuts perpendicular to the magnetic field direction, shifted in parallel velocity, in order to intersect the phase space region of maximum flux, which is observed at paral- lel velocities of the order or above $1000 \mathrm{~km} / \mathrm{s}$. The parallel velocity is close to the positive $Y$ direction. Let us first note that these observations are consistent with the model of an injection site at the shock. The observed dispersion is of the order of $80 \mathrm{~s}$, the injection site is thus at about $15 R_{E}$ from CLUSTER, which is a reasonable distance for a connection to the shock in the early morning side. In such a geometry, the injected particles must have a positive $V_{y}$ velocity to reach CLUSTER, which corresponds to the observation.

The distribution is very anisotropic in pitch-angles and displays a clear organization in ring with the central axis close to the magnetic field direction (close to the $Y$ direction in the present case). Typically, the aperture and the width of the ring are of the order of $45 \%$ and $30 \%$, respectively. In 3$\mathrm{D}$, the energetic particles are thus located in an hollow cone with an axis close to the magnetic field, their energy being limited by a minimum and maximum thresholds. Due to the energy/time dispersion, the energy thresholds decrease with time but the characteristics of the hollow cone (aperture and width) remain almost unchanged. More generally, we verify that MDS distributions are systematically ring-like. As discussed below, this type of distribution can be explained by considering the transmission of upstream particles across a quasi-perpendicular shock. Let us also note that the distribution presents some non-gyrotropic features. We have not studied in detail this aspect. It may indicate that the conditions of transmission across the shock are phase-angle dependent. This is also a typical feature of the particle interaction with a quasi-perpendicular shock. The organization of the accelerated particles in a ring-like distribution is the first constraint on the acceleration mechanism that we will consider. In Fig. 6, we present RAPID observations for the same event. They are spherical cuts of the distribution at energy, ranging from 30 to $60 \mathrm{keV}$. One again notices that the distributions display a ring-like feature. Futhermore, RAPID data indicate that the dispersed structures extend well above the CIS energy threshold. This is the second part of the puzzle: the acceleration mechanism that generates MDS must be efficient. Let us consider the model displayed in Fig. 4. The typical $X$ distance from CLUSTER to the front region of the shock, where the interaction begins, is $5 R_{E}$. Given the solar wind velocity, the drift of the discontinuity to CLUSTER position would be of the order of $100 \mathrm{~s}$. This is the maximum time period during which the acceleration may take place, according to the model. This can be compared to the ion gyroperiod in the solar wind. Taking a magnetic field of $5 \mathrm{nT}$, one obtains a gyroperiod of $16 \mathrm{~s}$, which shows that a significant acceleration must occur in a few gyroperiod only. The acceleration of charged particles at the shock as been the subject of many studies (see the reviews by Kirk, 1993; or Kuijpers, 1996, for example). For a quasi-perpendicular geometry (angle between the normal and the magnetic field larger than $45^{\circ}$ ), the main acceleration results from the action of the induced electric field during the particle drift in the shock magnetic gradient (shock drift acceleration, for a thorough list of references see the review paper by Armstrong et al., 1985). This mechanism leads to a fast (few gyroperiods) but limited ac- 

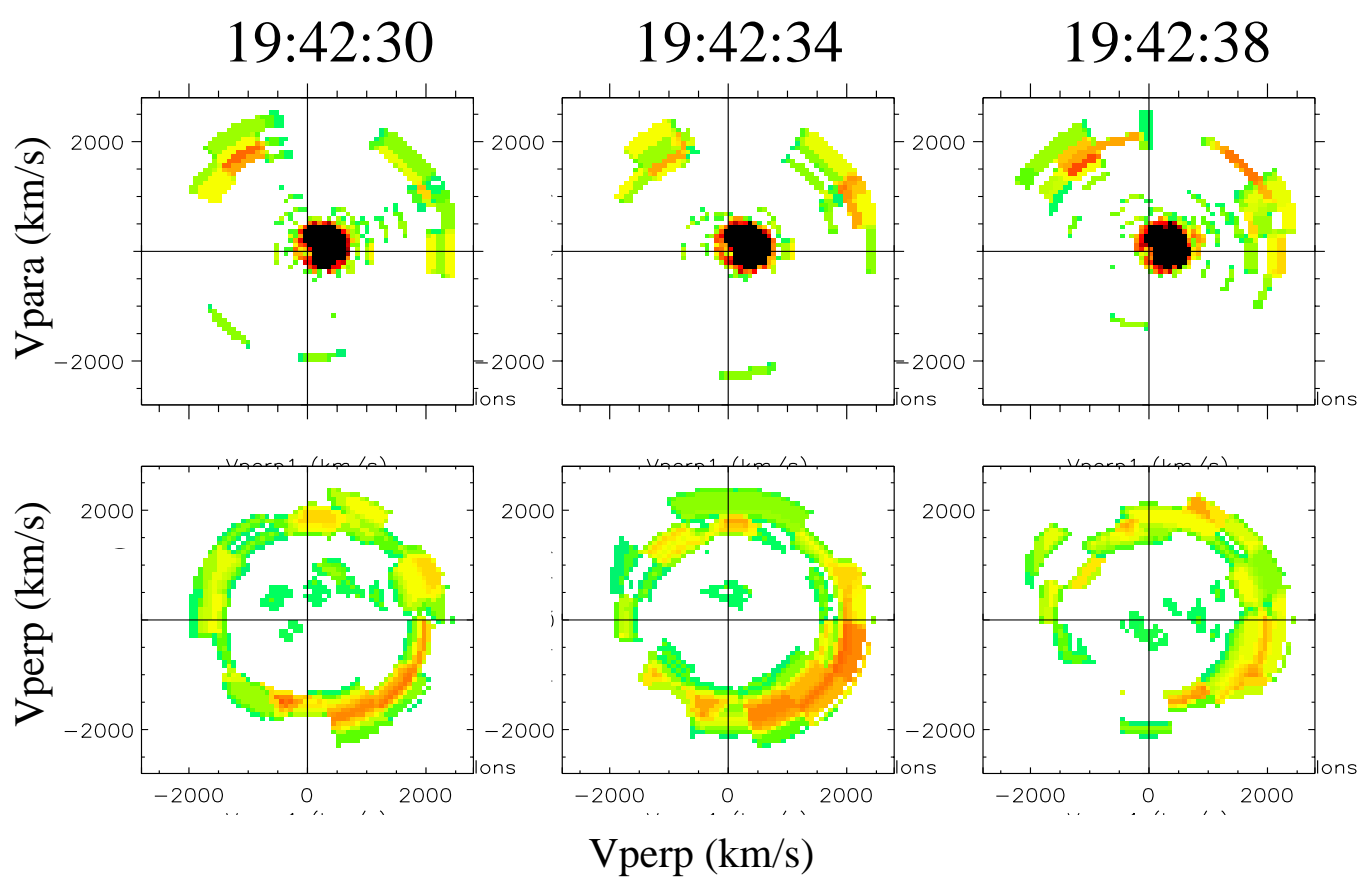

Fig. 5. Two-dimensinal cuts of the distribution function of the dispersed structure observed on 28 January 2001. The upper plots correspond to pitch-angle distributions. The lower plots are cuts in a plane perpendicular to the magnetic field shifted by $1000 \mathrm{~km} / \mathrm{s}$ along the parallel direction.

celeration, since the magnetic moment is approximately conserved during the process (Pesses, 1981). The acceleration is thus limited to a few times the initial particle energy. This mechanism corresponds to a weak scattering case, the conditions of transmission and reflection being pitch-angle and even phase-angle dependent. Quasi-perpendicular shocks thus lead to the reflection of nearly field-aligned ion beams and to the transmission of widely opened hollow cone distributions (Decker, 1983). For quasi-parallel shocks (angle between the normal and the magnetic field less than $45^{\circ}$ ), large amplitude magnetic fluctuations, both upstream and downstream the shock, with converging velocities, have been observed. It has been proposed that these fluctuations scatter the particles, leading to a Fermi acceleration (Bell, 1978); see the review by Scholer (1985). This mechanism is slower than the shock drift acceleration but it is not limited in energy. It corresponds to a strong scattering case; the diffuse acceleration thus leads to the formation of an isotropic population of high energy ions with a rather flat spectra (Thomsen, 1985).

One immediately notes the difficulty of reconciling the observations reported here with the existing theories. Shock drift acceleration would explain the observed pitch angle dependence but not the high energy of the accelerated particles. The diffuse acceleration would not be adequate for exactly inverse reasons!

In Fig. 7, we propose a model that combines the particle interaction with a quasi-perpendicular shock and a Fermi process linked to the arrival of a solar wind discontinuity. We will not make its detailed numerical analysis here. It indeed involves 3-D geometry effects, the complex physics of the particle interaction with the shock and the role of the solar wind discontinuity, which is beyond the scope of the paper. We rather propose to use elements already published, to develop a feeling for a possible acceleration mechanism resulting from the combined action of these different elements. In the first panel of Fig. 7, we display the general geometry of the interaction between the shock and the discontinuity in the plane perpendicular to the magnetic field in the discontinuity $\left(B_{d}\right)$. We consider the geometry of the 28 January event. We note that $B_{d}$ and the magnetic field in solar wind $\left(B_{s w}\right)$ are approximately perpendicular. $B_{s w}$ is thus in the plane of the figure. We show projections of typical ion trajectories in this plane. They are circles in the discontinuity, and projections of helix in the solar wind. Close to the shock nose (first contact point between the discontinuity and the shock), the shock geometry is quasi-perpendicular. In the panel below, we present a sketch of the expected consequences of the shock/particle interactions. From published works (Decker, 1983), one knows that in this region of the bow shock, a part of the incoming ions are reflected upstream to form a quite narrow beam in the solar wind magnetic field direction. In the downstream region, the transmitted ions have, on average, a large pitch angle. In phase space, they are thus located on a cone of large aperture and symmetric from the magnetic field. Numerical studies are needed to estimate the probability for a particle to be reflected or transmitted across the shock. From Armstrong, (1985), Fig. 7, we consider that a probability of transmission of 0.2 is reasonable.

The principle of the Fermi interaction is displayed in 

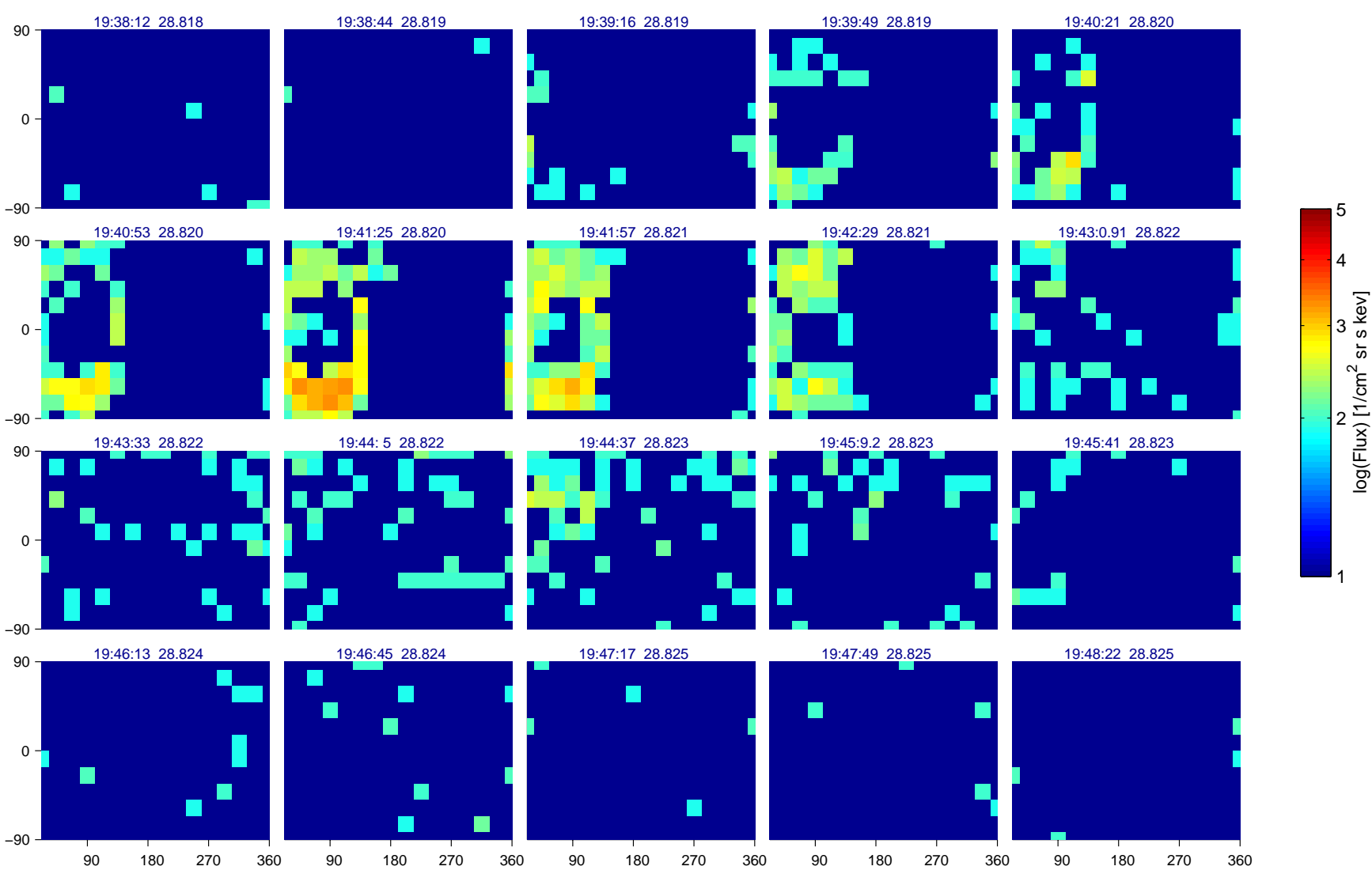

Fig. 6. Azimuthal and polar representations of energetic particle distributions measured by RAPID (30-60 keV).

Fig. 8. We display some expected trajectories; the general idea is that particles of the reflected beam gyrate in the discontinuity and may be re-injected toward the shock. The velocity increment that results from this reflection is typically of the order of $V_{s w}(300 \mathrm{~km} / \mathrm{s})$. If multiple interactions take place, the particles are then accelerated by a Fermi process. Just as an example, three interactions (gain of $900 \mathrm{~km} / \mathrm{s}$ ) would be sufficient to accelerate particles from $5 \mathrm{keV}(900 \mathrm{~km} / \mathrm{s})$ to $20 \mathrm{keV}(1800 \mathrm{~km} / \mathrm{s})$. Starting with a maxwellian at $1 \mathrm{keV}, 4-5$ interactions would be thus sufficient to produce high fluxes in the $20-30 \mathrm{keV}$ range. The gyration time is a fraction of gyroperiod (then a few seconds for a $5 \mathrm{nT}$ field). For particles bouncing close to the shock/discontinuity intersection, the transit time to the shock would be a couple of seconds. Thus, one estimates that 10$20 \mathrm{~s}$ are sufficient for a complete cycle (reflection at shock, gyration in the discontinuity and new interaction at shock). Five interactions may occur in $100 \mathrm{~s}$ which is the estimated time for the drift along the shock. We conclude that particles may be accelerated from $1 \mathrm{keV}$ to $20-30 \mathrm{keV}$ in a few tens of seconds in a restricted region of the front of the shock (not farther than $5 R_{E}$ from the nose).

Let us estimate the fraction of particles that could be accelerated. Using results of numerical simulations, the fraction of reflected particles in the beam has been estimated to be of the order of 0.4 (Armstrong, 1985). From an analysis of the reflection by the discontinuity, one deduces the equation that links the exit phase angle $\theta_{1}$ with the initial phase angle $\theta_{0}$, the particle velocity $\left(V_{0}\right)$ and the discontinuity velocity $\left(V_{d}\right)$. For $V_{0} \gg V_{d}$, one simply obtains:

$\theta_{1}=\pi \frac{V_{d}}{V_{0}}-\theta_{0}$

Assuming that the initial beam has a $30^{\circ}$ aperture $\left(60^{\circ}>\right.$ $\theta_{0}>90^{\circ}$ ), taking $V_{0}=4 V_{d}$ (which corresponds to $10 \mathrm{keV}$ ), one realizes that the exit angle is in the cone defined by: $-37^{\circ}>\theta_{1}>-67^{\circ}$. This phase angle is also the pitch angle of the particle in the region bounded by the discontinuity and the shock. To directly re-encounter the shock, the distance between the exit position and the shock must be smaller than typically 2 Larmor radii, otherwise, the particle has a free motion and escapes from the shock. Most of the particles that exit the discontinuity at less than 2 Larmor radii from the shock then re-encounter the shock and a new interaction takes place. Taking a reflection efficiency of 0.4 , the fraction of particles that may undergo 5 interactions would be of the order of $1 \%$. 


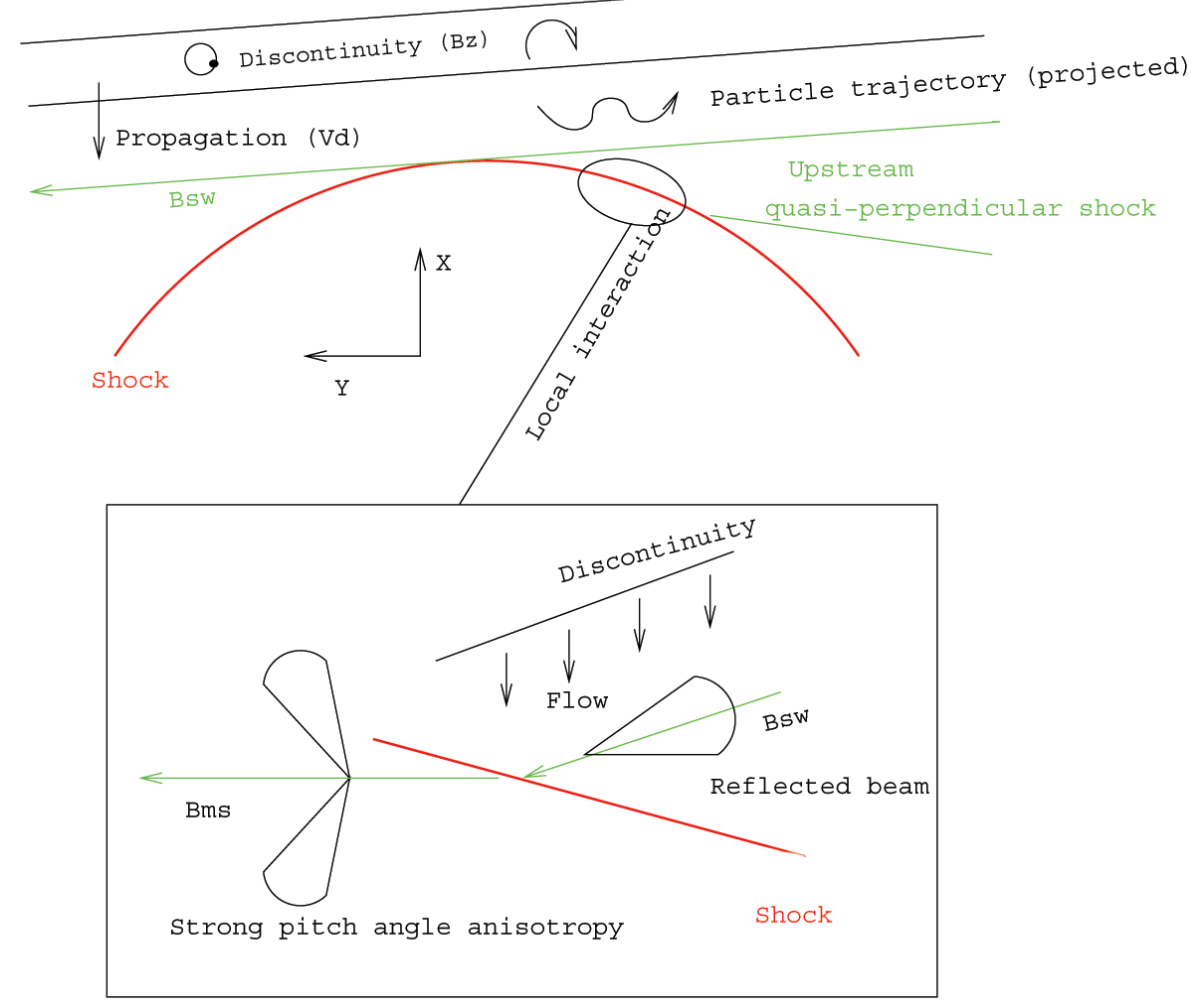

Fig. 7. General geometry of the interaction.

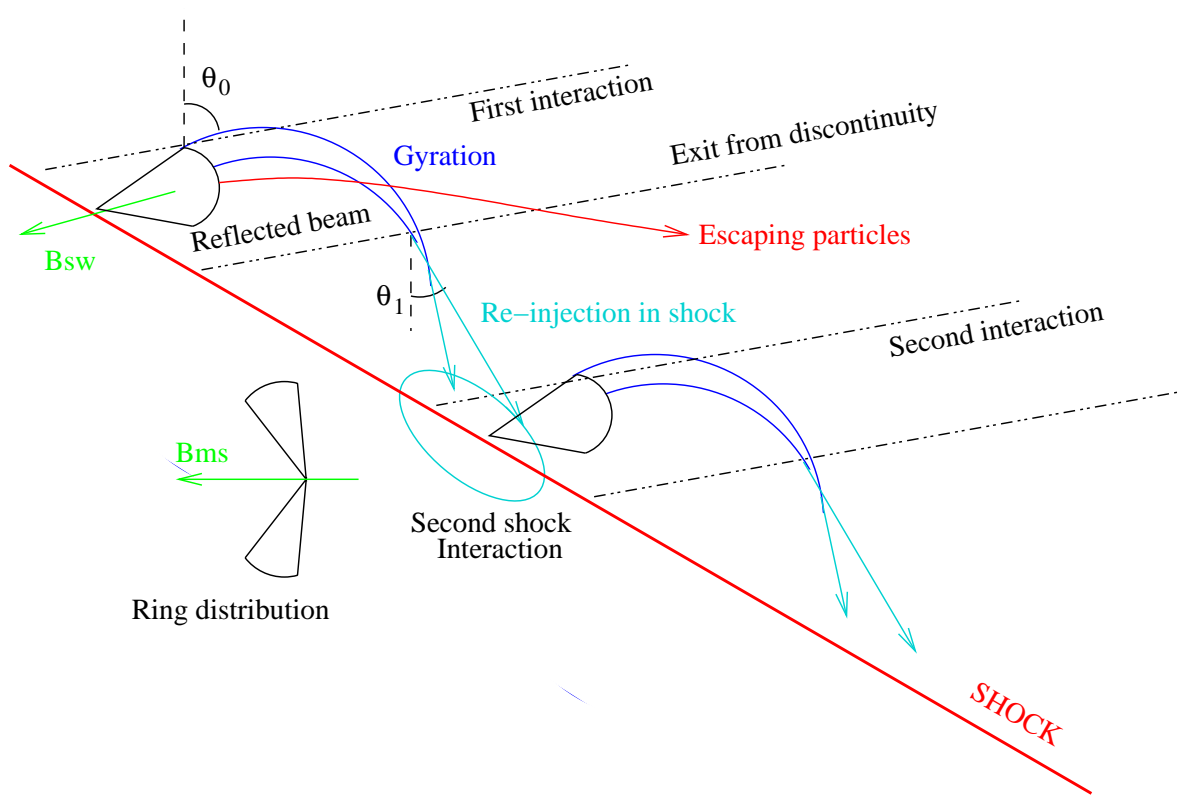

Fig. 8. A sketch of the Fermi acceleration of the reflected ion beam at quasi-perpendicular shock.

To support the model, one may note that the accelerated particles come only from one side of the shock (the negative $Y$ side in the present case). The model simply explains this particularity. The Fermi process may only develop at the side where the particle gyration tends to increase the dis- tance from the shock. Given the direction of the $z$ component of magnetic field in the discontinuity (ACE measures a positive $B_{z}$ ), the gyration is associated with a drift along the $-Y$ direction. It is thus in the $-Y$ side of the shock that the acceleration occurs. 
From this discussion, we conclude that a Fermi acceleration resulting from multiple interactions of the particles between the discontinuity and the shock is a possible model for the formation of MDS. The fast acceleration would be linked to the Fermi process (bouncing between the discontinuity and the shock) and the pitch angle selection of the transmitted particles would be associated with the physics of the interactions with the quasi-perpendicular shock.

A last point can be noticed. The Fermi process develops only for a particle that bounces close to the intersection point between the discontinuity and the shock. Particles that are reflected from the shock but interact with the discontinuity at larger distance will drift along the discontinuity, a situation quite similar to the model proposed by Burgess (1989a) for explaining the formation of HFA. The existence of two possible types of interactions (bouncing and drifting) could explain why MDS and HFA are often associated. When a discontinuity interacts with the shock, we propose that a small fraction of particles may bounce between the shock and the discontinuity, in a thin region just ahead of the shock/discontinuity intersection. These particles are Fermi accelerated. Some of them, transmitted across the shock, then reach the observer located in the magnetosheath and form the MDS. Other particles, with different initial conditions, drift along the discontinuity and progressively gain energy (drift acceleration). According to simulations, they can be thermalized and a hot plasma region is thus expected to be formed in the region behind the shock/discontinuity intersection. This would correspond to the formation of the HFA. This hot plasma could then be convected downstream at the magnetosheath velocity. Both the low energy part of the MDS and the hot turbulent plasma that characterized the HFA would thus reach the observer almost simultaneously. In such a picture, the MDS would be just a precursor of a HFA-type phenomena. This is precisely what is observed in Figs. 2 and 3.

\section{Conclusion}

We have reported observations of energy/time dispersed signatures made by the CLUSTER-CIS instrument in the magnetosheath. These signatures, characterized by significant increases in the flux of particles at energy higher than $10 \mathrm{keV}$, last typically 1-2 min. They are often associated with HFAtype phenomena, thus with solar wind discontinuities that perturb the shock thermalization processes. Analyzing the characteristics of the energy/time dispersion of a particularly well-defined example, we conclude that it is associated with an injection site that drifts along the shock. This suggests that its origin is a local and transient modification of the shock acceleration regime resulting from its interaction with a solar wind discontinuity. Nevertheless, we also report on the observations of a dispersed signature made close to a boundary layer. In such cases, their possible magnetospheric origin has to be considered.
The dispersed structures do not lead to significant pressure disturbances in the magnetosheath. Thus, they probably have a minor role in the solar wind/ magnetosphere coupling; their interest is elsewhere. In case of a shock origin, from the study of the distribution functions of the energetic particles, we deduce that a very specific acceleration process must operate at the shock. It combines features of the Fermi acceleration (acceleration to high energies) and of the shock drift acceleration (pitch angle selection of transmitted particle). A simple model of a Fermi acceleration taking place between a discontinuity that converges towards a quasi-perpendicular shock qualitatively explains the observations. The Fermi process greatly enhances the efficiency of the shock acceleration in energy, flux and, time. These observations directly prove the important role that may have shock/shock or discontinuity/shock collisions for producing large fluxes of accelerated particles. This has obvious applications in a more general astrophysical context.

Acknowledgements. The French part of this work has been supported by CNES and CNRS.

Topical Editor T. Pulkkinen thanks D. Winske and another referee for their help in evaluating this paper.

\section{References}

Armstrong, T. P., Pesses, M. E., and Decker, R. B.: Shock drift acceleration, in Collisionless shocks in the heliosphere: reviews of current research, ed. Tsurutani and Stone, Geophysical Monograph, 35, 271, 1985.

Arnoldy, R. L. and Chang, K. W.: Particle substorms observed at the geostationary orbit, J. Geophys. Res., 74, 5019, 1969.

Balogh, A., Dunlop, M. W., Cowley, S. W. H., et al.: The Cluster magnetic field investigation, Space Sci. Rev., 79, 65, 1997.

Bell, A. R.: The acceleration of cosmic rays in shock fronts, Mon. Not. R. Astron. Soc., 182, 147, 1978.

Burgess, D.: Cyclic behavior of quasi-parallel collisionless shocks, Geophys. Res. Lett., 16, 345, 1989a.

Burgess, D.: On the effect of a tangentially discontinuity on ions specularly reflected at an oblique shock J. Geophys. Res., 94, 472-478, 1989b.

Carlson, C. W. and Torbert, R. B.: Solar wind ion injections in the morning auroral oval, J. Geophys. Res., 85, 2903, 1980.

Crooker, N. U. and Siscoe, G. L.: A mechanism for pressure anisotropy and mirror instability in the dayside magnetosheath, J. Geophys. Res., 82, 185, 1977.

Decker, R. B.: Formation os shock-spike events at quasiperpendicualr shocks, J. Geophys. Res., 88, 9959, 1983.

DeForest, S. E. and Mcllwain, C. E.: Plasma clouds in the magnetosphere, J. Geophys. Res., 76, 3587, 1971.

Entgebretson, M. L., Lin, N., Baumjohann, W., Lühr, H., Anderson, B. J., Zanetti, L. J., Potemra, T. A., McPherron, R. L., and Kivelson, M. G.: A comparison of ULF fluctuations in the solar wind, magnetosheath, and dayside magnetosphere, 1 - Magnetosheath morphology, J. Geophys. Res., 96, 3441, 1991.

Fazakerley, N. A. and Southwood, D. J.: Mirror instability on the magnetosheath, Adv. Space Res., 14, 65, 1994.

Greenstadt, E. W.: Observation of nonuniform structure of the Earth's bow shock correlated with interplanetary field orientation, J. Geophys. Res., 77, 1729-1738, 1972. 
Hubert, D., Perche, C., Harvey, C. C., Lacombe, C., and Russell, C. T.: Observation of mirror waves downstream of a quasiperpendicular shock, Geophys. Res. Lett., 16, 159-162, 1989.

Kennel, C. F., Edmiston, J. P., Scarf, F. L., et al.: Structure of 12 November 1978, quasi parellel interplanetary shock, J. Geophys. Res., 89, 5436-5452, 1984.

Kirk, J. G.: Particle Acceleration, in Plasma astrophysics, ed. Benz and Courvoisier, Springer-Verlag, 1994.

Konradi, A., Semar, C. L., and Fritz, T. A.: Substorm-injected protons and electrons and the injection boundary model, J. Geophys. Res., 88, 543, 1975.

Kuijpers, J.: Particle Acceleration, in Plasma astrophysics, ed. Chiuderi and Einaudi, Springer-Verlag, 1996.

Lacombe, C., Pantellini, F. G. E., Hubert, D., Harvey, C. C., Mangeney, A., Belmont, G., and Russell, C. T.: Mirror and Alfvénic waves observed by ISEE1-2 during crossinds of the Earth's bow shock, Ann. Geophysicae, 10, 772, 1992.

Mauk, B. H. and Meng, C.-I.: Dynamic injections as the source of near geostationary quiet time particle spatial boundaries, J. Geophys. Res., 88, $10011,1983$.

Paschmann, G., Haerendel, G., Sckopke, N., Möbius, E., Lühr, H., and Carlson, C. W.: Thress-dimensional plasma structures with anomalous flow directions near the Earth's bow shock, J. Geophys. Res., 93, 11 279-11 294, 1988.

Pesses, M. E.: On the conservation of the first adiabatic inveriant in perpendicular shocks, J. Geophys. Res., 86, 150, 1981.

Rème, H., Bosqued J. M., Sauvaud J.-A., et al.: The Cluster ion spectrometry (CIS) experiment, Space Sci. Rev., 79, 303, 1997.

Sandahl, I., Koskinen, H. E., Mälkki, A. M., Pulkinen, T. I., Budnil, E. Y., Fedorov, A. O., Frank, L. A., and Sigwarth, J. B.: Dispersive magnetosheath-like ion injections in the evening sector on 11 January 1997, Geophys. Res. Lett., 25, 2568, 1998.

Sauvaud, J.-A., Crasnier, J., Mouala, K., Kovrazhkin, R. A., and Jorjio, N. V.: Morning sector ion precipitation following substorm injections, J. Geophys. Res., 86, 3430, 1981.

Scholer, M.: Diffusive acceleration, in: Collisionless shocks in the heliosphere: reviews of current research, (ed) Tsurutani and Stone, Geophysical Monograph 35, 287, 1985.

Schwartz, S., Chaloner, C. P., Christiansen, P. J., et al.: An active current sheet in the solar wind, Nature, 318, 269-271, 1985.

Schwartz, S. J., Kessel, R. L., Brown, C. C., Woolliscroft, L. J. C., Dunlop, M. W., Farrugia, C. J., and Hall, D. S.: Active current sheets near the Earth's bow shock, J. Geophys. Res., 93, 11 295$11310,1988$.
Schwartz, S.: Hot flow anomalies near the Earth's bow shock, Adv. Space Res., 15, 107-116, 1995.

Scudder, J. D., Burlaga, L. F., and Greenstadt, E. W.: Scale lengths in quasi-parallel shock, J. Geophys. Res., 89, 7545-7550, 1984.

Shue, J.-H, Song, P., Russell, C. T., Steinberg, J. T., Chao, J. K., Zastenker, G., Vaisberg, O. L., Kokubun, S., Singer, H. J., Detman, T. R., and Kawano, H.: Magnetopause location under extreme solar wind conditions, J. Geophys. Res., 103, 17 691-17 700, 1998.

Sibeck, D. G., Borodkova, N. L., Zastenker, G. N., Romanov, S. A., and Sauvaud, J.-A.: Gross deformation of the dayside magnetopause, Geophys. Res. Lett., 25, 453, 1998.

Sibeck, D. G., Borodkova, N. L., Schwarta, S. J., et al.: Comprehensive study of the magnetospheric response to a hot flow anomaly, J. Geophys. Res., 104, 4577-4593, 1999.

Song, P. and Russell, C. T.: What do we really know about the magnetosheath? Adv. in Space Res., 20, 747-765, 1997.

Stenuit, H., Sauvaud, J.-A., Delcourt, D. C., Mukai, T., Kokobun, S., Fujimoto, M., Buzulukova, N. Y., Kovrazhkin, R. A., Lin, R. P., and Lepping, R. P.: A study of ion injections at the dawn and dusk polar edges of the auroral oval., J. Geophys. Res., 106, $29619,2001$.

Thomas, V. A., Winske, D., and Omidi, N.: Re-forming supercritical quasi-parallel shocks 1 . One- and two-dimensional simulations, J. Geophys. Res., 95, 18 809, 1990.

Thomsen, M. F.: Upstream suprathermal ions, in: Collisionless shocks in the heliosphere: reviews of current research, (ed) Tsurutani and Stone, Geophysical Monograph 35, 253, 1985

Thomsen, M. F., Gosling, J. T., Fuselier, S. A., Bame, S. J., and Russell, C. T.: Hot diamagnetic cavities upstream from the Earth's bow shock, J. Geophys. Res., 91, 2961-2973, 1986

Thomsen, M. F., Gosling, J. T., Bame, S. J., Quest, B., Russell, C. T., and Fuselier, S. A.: On the origin of hot diamagnetic cavities near the Earth's bow shock, J. Geophys. Res., 93, 11 311-11 325, 1988

Thomsen, M. F., Thomas, V. A., Winskee, D., Gosling, J. T., Farris, M. H., and Russel, C. T.: Observational test of hot flow anomaly formation by the interaction of a magnetic discontinuity with the bow shock, J. Geophys. Res., 98, 15319-15330, 1993.

Winske, D., Omidi, N., Quest, K. B., and Thomas, V. A.: Reforming supercritical quasi-parallel shocks 2. Mechanism for wave generation and front re-formation, J. Geophys. Res., 95, $18821,1990$. 